\title{
Prevalencia de anticuerpos contra dengue y leptospira en la población de Jáltipan, Veracruz
}

\author{
Joel Navarrete-Espinosa, MD,(I) Juan Antonio Acevedo-Vales, MD,(I) Emilia Huerta-Hernández, QBP,(I) \\ Jorge Torres-Barranca, PhD, (2) Dolores Guadalupe Gavaldón-Rosas, MC. ${ }^{(2)}$
}

\section{Navarrete-Espinosa J,Acevedo-Vales JA, Huerta-Hernández E, Torres-Barranca J, Gavaldón-Rosas DG. Prevalencia de anticuerpos contra dengue y leptospira en la población de Jáltipan, Veracruz. Salud Publica Mex 2006;48:220-228.}

\section{Resumen}

Objetivo. Conocer la prevalencia de anticuerpos contra dengue y leptospira en Jáltipan,Veracruz, México. Material y métodos. Estudio transversal en $\mathbf{5 0 0}$ habitantes de Jáltipan, Veracruz, mediante un muestreo por hogares, aplicación de cuestionario y toma de muestras sanguíneas. Se buscaron anticuerpos $\lg G$ y se evaluaron factores asociados a sus seroprevalencias mediante regresión logística. Resultados. La seroprevalencia global para denguevirus fue 79.6\% (IC95\% 76-8I); los principales factores vinculados con la infección fueron la edad $(p<0.001)$ y presencia de larvas en el domicilio (RMP $=2.94 ;$ IC95\% I.46-6.I). La seroprevalencia global contra leptospira fue 4\% (IC95\% 2-6). La mayor prevalencia fue para el grupo en edad productiva $(35 \%$, IC95\% |5-59). Quienes refirieron convivir simultáneamente con perros, cerdos, vacas y ratas presentaron una seroprevalencia de $25 \%$ (IC95\% 0.63-80). El $85 \%$ de los positivos a leptospira también fue positivo a dengue. Conclusiones. La prevalencia de anticuerpos contra denguevirus demuestra el riesgo de brotes explosivos de dengue hemorrágico. Aunque la prevalencia de leptospirosis fue baja, se confirmó la coexistencia de estos agentes.

Palabras clave: dengue; leptospira; anticuerpos; prevalencia; México
Navarrete-Espinosa J,Acevedo-Vales JA, Huerta-Hernández E, Torres-Barranca J, Gavaldón-Rosas DG.

Prevalence of dengue and leptospira antibodies

in the state of Veracruz, Mexico.

Salud Publica Mex 2006;48:220-228.

\section{Abstract}

Objective.To discover the prevalence of antibodies against denguevirus and leptospira. Material and Methods. A cross-sectional study was carried out in 500 inhabitants of Veracruz, Mexico through household sampling, administration of a questionnaire and collection of blood samples. The presence of $\lg G$ antibodies was determined and factors associated with seroprevalence were evaluated using a logistic regression model. Results. Total denguevirus seroprevalence was 79.6\% (IC95\% 76-8I). The main factors associated with infection were age and residence in neighborhoods with higher vectorial indices (RMP $=2.94$, IC95\% I.46-6.I). Overall seroprevalence against leptospira was $4 \%$ (IC95\% 2-6). Prevalence was higher in the 25 to 44 years age group (35\%, IC $95 \%$ I5-59). Seroprevalence in individuals who referred to living with dogs, pigs, cows and rats was $25 \%$ (IC95\% 0.63-80). Eighty-five percent of individuals who were leptospira positive were also dengue positive. Conclusions. The prevalence of denguevirus antibodies suggests the risk of serious dengue hemorrhagic outbreaks. Although leptospirosis prevalence was low, the coexistence of both agents was confirmed.

Key words: dengue; leptospira; antibodies; prevalence; Mexico

(I) Coordinación de Programas Integrados de Salud, Unidad de Salud Pública, Instituto Mexicano del Seguro Social, México, DF.

(2) Laboratorio de Leptospira, Universidad Autónoma Metropolitana, Xochimilco, México, DF.

Fecha de recibido: 9 de junio de 2005 - Fecha de aceptado: 6 de marzo de 2006 Solicitud de sobretiros: Joel Navarrete Espinosa, División de Epidemiología. Coordinación de Programas Integrados de Salud (IMSS). Av. Insurgentes Sur 253-2 Piso, col. Roma Sur, 06700 México, México.

Correo electrónico: joel.navarrete@imss.gob.mx, jonavaes@salud.gob.mx 
L os cambios climáticos observados durante los últimos años en todo el planeta han repercutido en forma sustancial en la modificación de los nichos ecológicos en que se desarrollan muchas de las enfermedades infecciosas, en especial aquellas que son transmitidas por vector y las zoonosis, lo que plantea el riesgo de un incremento en la ocurrencia de algunas como el dengue, y la reaparición y diseminación de otras como las encefalitis virales, la fiebre amarilla y la leptospirosis. ${ }^{1-4}$ En la actualidad se reconoce que el dengue es un importante problema de salud pública en el mundo, ya que durante los últimos años el número de casos comunicados se incrementó y de manera anual la cifra de enfermos asciende a millones, con una cantidad considerable de casos de dengue hemorrágico y de muertes por esta causa. .-8 $^{-8}$

México no es ajeno a este problema y hoy en día reúne las condiciones geográficas, ambientales y socioeconómicas $^{9,10}$ que propician la ocurrencia de brotes importantes de dengue hemorrágico, así como la transmisión de la enfermedad en regiones que antes no se consideraban propicias. ${ }^{11} \mathrm{Su}$ comportamiento epidemiológico ha mostrado variaciones en las tasas de incidencia, con una tendencia negativa hasta 1994, y, a partir de 1995, con la diseminación del vector a la mayor parte del territorio nacional y los altos índices vectoriales registrados ${ }^{12,13}$ se asiste a un incremento explosivo en el número de notificaciones y entidades federativas afectadas. No sólo eso, sobre todo llama la atención la cada vez más frecuente notificación de formas hemorrágicas graves, relacionadas con mucha probabilidad con el antecedente de la circulación de los cuatro denguevirus y con la circulación simultánea de dos o más de éstos en algunas entidades, ${ }^{14-18}$ de forma tal que en los días que corren se encuentran zonas consideradas como endémicas, en donde la transmisión es casi permanente. Este hecho se hace patente al observar la distribución geográfica de la enfermedad en el país durante los primeros años de transmisión cuando sólo se había identificado la circulación del Den-1 y el grado de diseminación alcanzado hasta la fecha una vez confirmada la circulación de los cuatro denguevirus (figuras 1 y 2). La situación se vuelve más ilustrativa si se compara el número de casos de dengue clásico y hemorrágico confirmados en todo el país (51 406 y 0, respectivamente) y en el estado de Veracruz (3 061 y 0, respectivamente) durante 1980 con los registrados durante el 2003 en el país (5 220 y 1 776, respectivamente) y ese estado (1 084 y 221, respectivamente). ${ }^{19,20}$

En este sentido, el análisis de la información generada por el Sistema de Vigilancia Epidemiológica Institucional ha demostrado en algunas regiones un

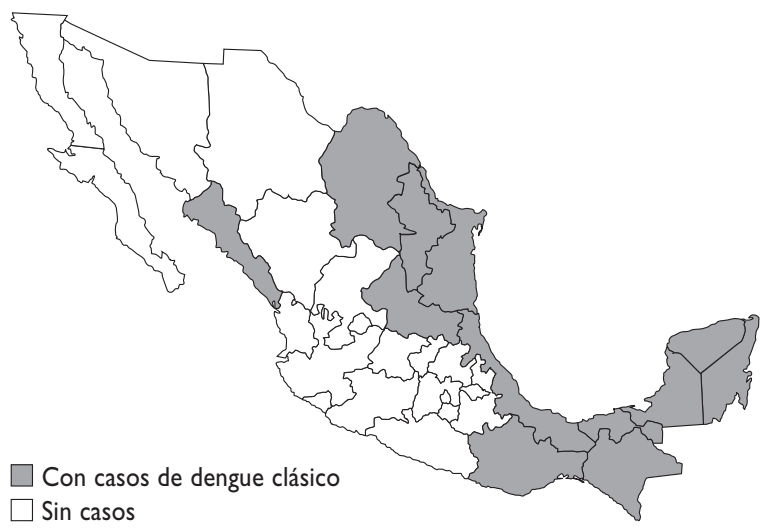

Figura I. Delegaciones con dengue en la República Mexicana (I978-I98I)

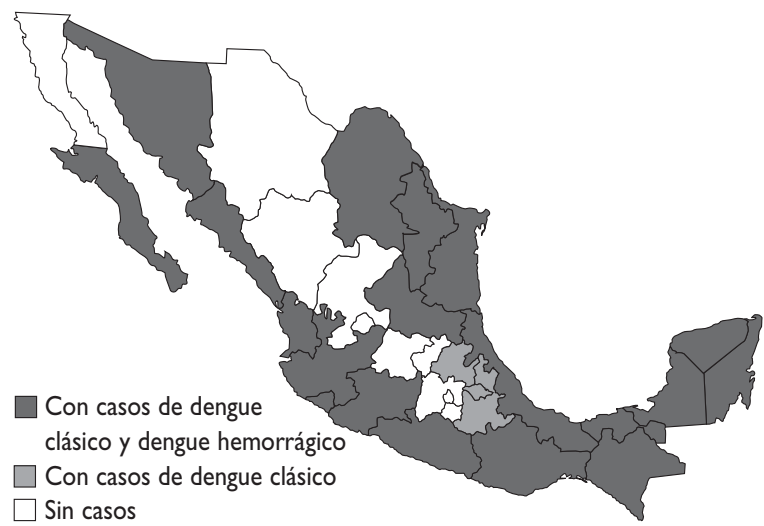

Figura 2. Delegaciones con dengue en la República Mexicana (2000-2003)

incremento de notificaciones de pacientes inicialmente diagnosticados con dengue que resultan negativos a las pruebas confirmatorias para esta infección, quienes al ser estudiados, en algunos casos, resultan positivos a leptospira. ${ }^{21}$ La similitud con que se presentan ambas enfermedades en su fase inicial ha propiciado dificultades en el nivel operativo para el diagnóstico oportuno y la atención adecuada de los enfermos, así como para su notificación y clasificación.

La coexistencia de diversos agentes patógenos causantes de enfermedad febril y hemorrágica como el dengue obliga a tratar de identificarlos y conocer el nivel de transmisión de cada enfermedad, así como los aspectos clínicos que comparten o las hacen diferentes para 
poder estimar la magnitud real del problema y ofrecer al médico herramientas que le permitan establecer un manejo específico oportuno.

El estado de Veracruz posee las condiciones ecológicas propicias para la transmisión de muchas enfermedades y, de acuerdo con el número de notificaciones, es hasta la fecha una de las entidades considerada de alta endemicidad para dengue, en donde además se ha observado la aparición de casos de leptospirosis durante los últimos años; no obstante, pese a que la notificación de esta última es cada vez más frecuente, son pocos los estudios realizados en el ámbito nacional y en el estado que permitan establecer un marco de referencia adecuado para la enfermedad. Por ejemplo, resta por establecerse la proporción de habitantes que han sido infectados por ambos agentes en una localidad endémica.

\section{Material y métodos}

Se realizó un estudio de prevalencia de anticuerpos en la población cubierta por el Programa IMSS-Oportunidades en Jáltipan, Veracruz, durante el año 2003. Considerando una prevalencia de anticuerpos contra dengue en la población de $30 \%$, un poder de la prueba de $80 \%$, nivel de confianza de $95 \%$ y una pérdida estimada de $15 \%$, se estimó un tamaño muestral de 500 individuos que fueron seleccionados mediante muestreo aleatorio en cada hogar. Se eligieron personas de cualquier edad y sexo con residencia permanente en la localidad. Previo consentimiento informado y firmado, a cada participante se le aplicó un cuestionario para obtener información personal y de factores de riesgo; en forma paralela y durante la visita, un técnico entomólogo realizó la inspección de la vivienda en busca de criaderos potenciales para la determinación de los índices larvarios ${ }^{22}$ de recipiente (I.R.), casa (I.C.) $\mathrm{y}$ de Breteau (I.B.= recipientes positivos por casa); a su vez, se revisó la presencia de mosquiteros y pabellones. A cada participante se le tomó una muestra de sangre venosa para la determinación de anticuerpos, la cual se obtuvo en condiciones estériles y se conservó en refrigeración $\left(-20^{\circ} \mathrm{C}\right)$ hasta su proceso. De cada muestra se obtuvieron dos partes para la búsqueda de anticuerpos específicos. La determinación de IgG contra dengue se realizó en el laboratorio de la Coordinación de Programas Integrados de Salud utilizando ELISA $^{23,24}$ (UMELISA-TECNOSUMA: sensibilidad $98.7 \%$, especificidad $94.2 \%$ ) y la determinación de anticuerpos contra leptospira se realizó en el laboratorio de leptospira de la Facultad de Veterinaria de la Universidad Autónoma Metropolitana (Xochimilco) utilizando la técnica de aglutinación microscópica ${ }^{25}$ (sensibilidad 95\%, especificidad 90\%) para identificar 13 serovariedades de Leptospira interrogans, icterohaemorrhagiae (cepa RGA), hebdomadis, pyrogenes, grippotyphosa, canicola, pomona, hardjo (cepa Hardjo-parajitnio), wolffi, tarassovi, bratislava, Pórtland-vere (cepa Sinaloa ACR), hardjo (cepa H-89) e icterohaemorrhagiae (cepa Palo Alto), las tres últimas correspondientes a aislamientos realizados en México.

Se analizaron la edad y sexo de los encuestados, así como la colonia de residencia, estrato socioeconómico, conocimientos de la enfermedad, uso de medidas de protección específica (mosquiteros, pabellones, insecticida), presencia de larvas en el domicilio y la convivencia con animales domésticos. Se obtuvieron frecuencias simples y proporciones, medidas de tendencia central y dispersión. Asimismo, se estimó la prevalencia de anticuerpos contra el denguevirus y leptospira con intervalos de confianza al 95\% (IC95\%). En las variables cualitativas se utilizó ji cuadrada simple y de tendencias; asimismo, de acuerdo con su distribución, se utilizó prueba t de Student o prueba U de Mann Whitney para la comparación de las variables cuantitativas. Como medida de efecto se obtuvo la razón de momios de prevalencia (RMP; IC95\%). Finalmente, para encontrar un modelo que explicara mejor la presencia de anticuerpos (infección por estos agentes) se utilizó regresión logística no condicional. El análisis se realizó con el paquete estadístico SPSS.

\section{Resultados}

Se analizaron encuestas y muestras serológicas de 500 personas. El 41\% correspondió a hombres y el resto a mujeres. La edad promedio fue 29.1 años y la mediana 28. Los grupos de edad que predominaron en la muestra fueron el de 25 a 44 años (33\%) seguido por el de $15-24(19 \%)$, el de 5-14 (16\%) y el de 45-64 años (15\%).

Prevalencia de anticuerpos contra dengue. La seroprevalencia para denguevirus en la población general fue de $79.6 \%$ (IC95\% 76-81) y ligeramente mayor en las mujeres ( $81 \%$, IC95\% 76-85) que en los hombres $(78 \%$, IC95\% 72-84). Asimismo, se observó una relación directa con la edad, hasta llegar a $94 \%$ en los individuos con 65 o más años (cuadro I).

Aspectos sociodemográficos. En relación con el nivel socioeconómico no se encontraron diferencias significativas entre los tres niveles $(79,78.4$ y $79.9 \%$, respectivamente). En cuanto a la utilización de medidas de protección, las diferencias fueron mínimas. Sin embargo, de acuerdo con el nivel de conocimientos de la enfermedad, la prevalencia fue mayor entre los que demostraron un nivel inadecuado (87.2\%) en compa- 


\begin{tabular}{|c|c|c|}
\hline \multicolumn{3}{|c|}{$\begin{array}{l}\text { Prevalencia de anticuerpos (IgG) contra dengue } \\
\text { Y leptospira, Jáltipan, Veracruz, MéXICo, } 2003\end{array}$} \\
\hline Variables & $\begin{array}{c}\text { Dengue } \\
(n=500)\end{array}$ & $\begin{array}{l}\text { Leptospira } \\
(n=20)\end{array}$ \\
\hline \multicolumn{3}{|l|}{ Edad } \\
\hline$<1$ & $17(0.4-64)$ & 0 \\
\hline $1-4$ & $66(46-82)$ & $3(0.1-17)$ \\
\hline $5-14$ & $69(59-78)$ & $5(2-I I)$ \\
\hline $15-24$ & 79 (68-87) & $7(3-15)$ \\
\hline $25-44$ & $86(81-91)$ & $4(2-8)$ \\
\hline $45-44$ & $86(76-93)$ & I (0.I-7) \\
\hline $65 y+$ & 94 (73-99) & 0 \\
\hline
\end{tabular}

Nivel socioeconómico

\begin{tabular}{lll} 
Alto & $79(71-88)$ & $3(1.4-7)$ \\
\hline Medio & $78(73-84)$ & $4(1.5-7.2)$ \\
\hline Bajo & $80(75-85)$ & $6(2.3-13)$
\end{tabular}

Grado de conocimientos

\begin{tabular}{ll} 
Adecuado & $78(74-82)$ \\
\hline Inadecuado & $87(78-93)$
\end{tabular}

Medidas de protección

\begin{tabular}{lr}
0 & 100 \\
\hline 1 & $82(76-87)$ \\
\hline $2 y+$ & $79(74-85)$
\end{tabular}

Índice de Breteau

Control $77(73-81)$

Emergencia 90 (83-95)

Convivencia con animales

\begin{tabular}{lr} 
Perro & I $(0.1-5)$ \\
\hline Perro y gato & $3(0.8-7)$ \\
\hline Perro, cerdo, vacas y ratas & $25(0.6-80)$
\end{tabular}

( ) $1 \mathrm{C} 95 \%$

ración con los individuos de nivel adecuado (78\%) (cuadro I). Asimismo, la presencia de anticuerpos fue mayor en los individuos residentes de las colonias que presentaron los mayores índices vectoriales (cuadro II). Aspectos epidemiológicos. Las personas que refirieron haber padecido dengue en forma previa registraron una seroprevalencia de $85 \%$, mientras que los que negaron dicho antecedente presentaron una frecuencia de 79\%; de igual forma, quienes refirieron sintomatología de sospecha para dengue (fiebre, cefalea, dolor retroocular, mialgias o artralgias), independientemente del

\section{Cuadro II \\ Prevalencia de anticuerpos (IgG) contra dengue Y LEPTOSPIRA SEGÚN ÍNDICES VECTORIALES Y LUGAR de Residencia, Jáltipan, Veracruz, MéXico, 2003}

\begin{tabular}{lccc} 
Colonia & Dengue & Leptospira & Indice de Breteau \\
Ciénega & $95(75-99)$ & $15(3-38)$ & $25^{*}$ \\
\hline Xochitonapa & $91(77-98)$ & $6(1-19)$ & $20^{*}$ \\
\hline Ferrocarrilera & $88(75-95)$ & 0 & $18^{*}$ \\
\hline Ampl. deportiva & $85(68-95)$ & 0 & 9 \\
\hline Murillo Vidal & $80(70-8 I)$ & $2(0.2-7.7)$ & 12 \\
\hline Deportiva & $77(63-88)$ & $13(4.7-25)$ & 10 \\
\hline Benito Juárez & $75(40-97)$ & 0 & 0 \\
\hline Emiliano Zapata & $75(35-97)$ & 0 & 0 \\
\hline Agraria & $74(68-80)$ & $3(1.4-6.9)$ & 14 \\
\hline Isabel & $67(22-96)$ & 0 & 0
\end{tabular}

() $1 C 95 \%$

*Nivel de emergencia

recuerdo de haber enfermado de dengue en fecha anterior, presentaron una mayor prevalencia (88\%) comparados con los que negaron toda sintomatología (78\%, IC95\% 74-82).

Análisis bivariado. Al comparar la prevalencia de infección por denguevirus, no se observaron diferencias entre ambos sexos. Respecto a la edad, se comprobó una tendencia positiva importante (ji cuadrada de tendencia $=24.38, p<0.001$ ); tomando como referencia a los menores de 5 años de edad, los individuos de 15 a 24 años tuvieron una prevalencia casi tres veces mayor, las personas de 25 a 44 años presentaron cuatro veces más y los individuos con 45 o más años cinco veces mayor prevalencia (cuadro III). Al agrupar por edad y sexo persiste la tendencia conforme a la edad, aunque la relación fue mayor en el sexo femenino (ji cuadrada de tendencia $=19.01, p<0.001)$ que en el masculino (ji cuadrada de tendencia $=6.12, p=0.001$ ).

Respecto al extracto socioeconómico no se encontraron diferencias; empero, las personas que demostraron poco conocimiento de la enfermedad presentaron una prevalencia 92\% mayor en comparación con los que obtuvieron una buena calificación en los conocimientos. Por otro lado, en quienes refirieron utilizar una o ninguna medida de protección contra el vector la prevalencia fue mayor en comparación con los que recurrieron a tres o más medidas de protección (cuadro III).

De acuerdo con los índices larvarios encontrados en la vivienda, los habitantes de las colonias que tu- 


\begin{tabular}{|c|c|c|}
\hline & & \\
\hline FACTORE & A INFECCIÓN P & GUE; \\
\hline ANÁLISIS BII & N, VERACRUZ, & 2003 \\
\hline Variables & RMP (IC95\%) & $p$ \\
\hline Sexo & & \\
\hline Masculino & 1.0 & \\
\hline Femenino & I.I9 (0.75-I.88) & 0.44 \\
\hline Edad & & \\
\hline$<5$ & 1.0 & \\
\hline $5-14$ & $1.65(0.69-3.91)$ & 0.21 \\
\hline $15-24$ & $2.75(\mathrm{I} .08-7.0 \mathrm{I})$ & 0.17 \\
\hline $25-44$ & 4.64 (1.97-10.97) & 0.001 \\
\hline $45 y+$ & $5.19(1.93-14.15)$ & 0.001 \\
\hline
\end{tabular}

Nivel socioeconómico

\begin{tabular}{lll} 
Alto & 1.0 & \\
\hline Medio & $0.82(0.43-1.59)$ & 0.53 \\
\hline Bajo & $1.10(0.57-2.14)$ & 0.75
\end{tabular}

Grado de conocimientos

\begin{tabular}{lll} 
Bueno & 1.0 & \\
\hline Malo & $1.92(0.94-4)$ & 0.05
\end{tabular}

Medidas de protección

\begin{tabular}{lll}
3 y más & 1.0 & \\
\hline 2 & $1.25(0.66-2.36)$ & 0.46 \\
\hline I o ninguna & $1.49(0.76-2.90)$ & 0.21
\end{tabular}

Índice de Breteau

\begin{tabular}{lll} 
Control & 1.0 & \\
\hline Emergencia & $2.84(1.37-6.07)$ & 0.003
\end{tabular}

vieron grado de control en "emergencia" para el I.B. $(R M P=2.84 ; I C 95 \% 1.37-6.07)$, I.C. $(R M P=2.93 ; I C 95 \%$ 1.26-5.70) e I.R. ( $R M P=1.49$; IC95\% 0.51-4.2) presentaron mayor prevalencia en comparación con las que tuvieron grado de control "óptimo". Por el contrario, al evaluar el recuerdo de haber enfermado por dengue, no se encontraron diferencias en la prevalencia de quienes contestaron afirmativamente y quienes negaron el antecedente.

Análisis multivariado. Los factores que permanecieron en el modelo multivariado final ajustado por los años de exposición del individuo (edad) y que se relacionan fuertemente con la presencia de anticuerpos fueron el bajo conocimiento de la enfermedad y la presencia de altos índices larvarios en el domicilio (cuadro IV).

\begin{tabular}{|c|c|c|}
\hline \multicolumn{3}{|c|}{$\begin{array}{c}\text { FACTORES DE RIESGO PARA INFECCIÓN POR DENGUE; } \\
\text { ANÁliSIS MULTIVARIADO; JÁltIPAN, VERACRUZ, } \\
\text { MÉXICO, } 2003\end{array}$} \\
\hline Variables & RMP (IC95\%) & $p$ \\
\hline \multicolumn{3}{|l|}{ Edad } \\
\hline$<5$ & 1.0 & \\
\hline $5-14$ & $1.7(0.76-3.9)$ & 0.188 \\
\hline $15-24$ & $2.7(1.1-6.5)$ & 0.023 \\
\hline $25-44$ & $4.7(2.01-10.6)$ & 0.001 \\
\hline $45 y+$ & $5.9(2.4-15.1)$ & 0.001 \\
\hline \multicolumn{3}{|c|}{ Grado de conocimientos } \\
\hline Bueno & I & \\
\hline Malo & $1.88(0.90-4)$ & 0.05 \\
\hline \multicolumn{3}{|l|}{ Índice de Breteau } \\
\hline Control & 1 & \\
\hline Emergencia & $2.94(I .46-6 . I)$ & 0.003 \\
\hline
\end{tabular}

Prevalencia de anticuerpos contra leptospira. La prevalencia global fue de $4 \%$ (IC95\% 2-6), con un ligero predominio del sexo masculino sobre el femenino (4.9\%, IC95\% 2-9 y 3.4\%, IC95\% 2-6, respectivamente); las serovariedades identificadas fueron: tarassovi (en $50 \%$ de los casos positivos), bratislava (20\%), icterohaemorragiae: pomona, pyrogenes y Palo Alto (15\% respectivamente), así como grippotyphosa, H-89 y Sinaloa ACR (10\% respectivamente) y canícola (5\%). No se identificaron casos positivos para las cepas hebdomadis, hardjo y wolffi. Cinco individuos reaccionaron contra dos o más serovariedades (dilución 1:80 o mayor), y en dos de ellos se registró una fuerte reacción (1:160) para dos serovariedades (pomona y Sinaloa ACR; y bratislava y Palo Alto).

El grupo de 15 a 24 años de edad fue el más afectado con una prevalencia de $7 \%$, seguido por el de 5 a 14 años con $5 \%$, el de 25 a 44 años con $4 \%$ y los de 1 a 4 y 45 a 64 con 3 y $1 \%$, respectivamente. No se encontraron casos positivos en las personas menores de 1 año y en los de 65 o más años (cuadro I).

Antecedentes epidemiológicos. En relación con la presencia de animales domésticos, $1 \%$ de los que manifestaron convivir con perros presentó anticuerpos contra leptospira, cifra que se incrementó a 2.8\% cuando también había gatos y hasta $25 \%$ entre los que manifestaron la presencia de perros, vacas, cerdos y ratas en la vivienda (cuadro I). No se identificaron casos en quie- 
nes manifestaron convivir con otros animales. La serovariedad icterohaemorrhagiae se identificó en aquellos que cohabitaban con perros, en los que tenían perros y gatos fueron icterohaemorrhagiae y tarassovii, $\mathrm{y}$, finalmente, para los que conviven con perros, gatos, vacas, cerdos y ratas las serovariedades identificadas fueron bratislava, pyrogenes, pomona, Palo Alto, Sinaloa ACR y grippotyphosa.

La prevalencia fue mayor entre las personas que acostumbran bañarse en ríos o lagunas (2.6\%, IC $95 \%$ 0.7-7) comparadas con las que no observan dicha costumbre $(1.3 \%$, IC $95 \%$ 0.2-5). Asimismo, los que refirieron encharcamientos peridomiciliarios registraron una prevalencia de $4.8 \%$ (IC95\% 0.1-24), mientras que en los que no los refirieron fue de 1.7\% (IC95\% 0.6-4).

En relación con la presencia de anticuerpos contra ambas infecciones se encontró que sólo 76\% del total de encuestados presentó anticuerpos contra dengue y $0.6 \%$ contra leptospira, $3.4 \%$ fue positivo para dengue y leptospira, y el resto fue negativo a ambas pruebas. Asimismo, las colonias que presentaron la mayor prevalencia de anticuerpos contra leptospira también presentaron la mayor proporción de individuos positivos para dengue (cuadro II).

\section{Discusión}

Los resultados obtenidos confirman la intensidad de la transmisión del denguevirus en México, en el estado de Veracruz y en la ciudad de Jáltipan. La seroprevalencia encontrada ubica a esta población, y pudiera decirse que a toda la entidad, como una zona hiperendémica. Asimismo, considerando los datos informados en todo el país, ${ }^{14}$ demuestran un incremento importante en el número de personas que han sido expuestas a la infección; por otro lado, hacen evidente la transmisión de la leptospirosis, enfermedad no considerada en el marco epidemiológico de la región hasta hace unos años, en coincidencia con el desarrollo de graves inundaciones que han afectado al territorio mexicano durante la última década.

Dengue. Los factores de riesgo encontrados son consistentes con resultados de otros estudios realizados en México ${ }^{10,26}$ y en $\mathrm{Cuba},{ }^{27-29}$ en donde se registra, aunque no en forma significativa, una mayor prevalencia en el sexo femenino y un gradiente positivo conforme se incrementa la edad. Estos factores han sido atribuidos a los hábitos peridomiciliarios del mosquito que determinan una mayor exposición de las mujeres, así como al periodo de exposición prolongado en los individuos de mayor edad.

La literatura describe al mejoramiento ambiental y a la participación comunitaria activa como uno de los principales ejes para el control del vector y para limitar la transmisión de la infección; ${ }^{30}$ sin embargo, el éxito de las estrategias depende del cambio de conductas que ha de sustentarse en un conocimiento adecuado de la enfermedad y de las medidas preventivas. La protección que brinda el conocimiento de la enfermedad y el uso de medidas de protección contra el vector ha sido comprobada en diferentes estudios que encuentran menor riesgo de infección según el grado de escolaridad y el uso correcto de insecticidas. ${ }^{31}$ Por otro lado, el impacto de las intervenciones educativas ha sido demostrado en México y Puerto Rico ${ }^{32,33}$ al observarse una disminución de los criaderos potenciales del vector relacionada con el cambio de actitud posterior a una intervención educativa. Los resultados del presente estudio, aunque en forma indirecta, corroboran este hecho al demostrar que los individuos que presentaron un bajo nivel de información presentaron casi dos veces mayor riesgo de haberse infectado en comparación con aquellos que demostraron un conocimiento adecuado.

La medición de los índices vectoriales demostró la presencia de larvas en condiciones de alarma en 70\% de los hogares encuestados y en situación de emergencia en aproximadamente $30 \%$, lo que permite inferir altas densidades del vector en su forma adulta. En este sentido, aunque existen informes de la protección adquirida con el uso de insecticidas y mosquiteros, ${ }^{10,31}$ el alto grado de exposición que enfrenta la población de Jáltipan, así como el uso obligado de múltiples mecanismos para evitar ser picado, impidieron demostrar tal efecto protector, a lo que habría que agregar el uso oportuno o inadecuado de tales medidas.

Leptospira. La prevalencia encontrada de anticuerpos contra leptospira es baja si se consideran los resultados de estudios realizados en Chiapas $(37.7 \%)^{34}$ y Yucatán, ${ }^{21}$ aunque esta diferencia puede atribuirse al tipo de población estudiada. No obstante, existe congruencia con esta información y con la de otros países ${ }^{35}$ al encontrar una mayor proporción de individuos infectados en el sexo masculino y en los grupos de edad productiva, hecho por el que la enfermedad se relaciona con factores ocupacionales. Los casos encontrados en niños menores de cuatro años demuestran que en Jáltipan la infección puede adquirirse desde temprana edad. Estos hallazgos permiten afirmar que el grado de exposición y el riesgo de infectarse lo determina la convivencia con animales domésticos. En este sentido, las serovariedades identificadas con mayor frecuencia fueron aquellas que utilizan como hospederos al ganado bovino, ovino y porcino; ${ }^{36}$ para sorpresa de todos, pese a que una gran proporción de pobladores manifestó convivir con perros, sólo en un caso se iden- 
tificaron anticuerpos contra la serovariedad canícola, la que además presentó reacción para gryppotyphosa y pyrogenes. Las serovariedades identificadas y su frecuencia son semejantes a lo encontrado en la población de Chiapas y Yucatán, ${ }^{21,34,37,38}$ pero difieren de lo encontrado en otros estados en donde las variedades icterohemorrágica, pomona y canícola se identificaron con mayor frecuencia. 39,40

Un aspecto importante a considerar es la alta proporción de personas con anticuerpos contra leptospira que también resultaron positivas a dengue (85\%), lo que implica la coexistencia de ambas infecciones en el mismo nicho ecológico. Este aspecto se demuestra por el hecho de que coinciden altas prevalencias de ambos padecimientos en las mismas áreas de la localidad estudiada.

Limitaciones metodológicas. Como todos los estudios transversales, los resultados del presente se encuentran limitados por aspectos inherentes al diseño. ${ }^{41}$ Uno de los más importantes se relaciona con la ambigüedad temporal que se presenta al medir la relación entre el inicio de una exposición y el momento en que se presenta su efecto. En este caso, los riesgos relacionados con el conocimiento de la enfermedad, las medidas de protección utilizadas y los índices vectoriales están sujetos al supuesto de que existió un cambio de actitud en la población luego de que recibió información relacionada con la enfermedad, cuyo efecto fue la eliminación de criaderos y evitar los piquetes de insectos; asimismo, se supone que la utilización de medidas de protección como los mosquiteros e insecticidas se usan correctamente. Por otro lado, la medición de los índices larvarios se realizó al momento de la entrevista, otra vez bajo el supuesto de que estas mediciones reflejan en forma indirecta las densidades del vector adulto y una situación que precedió el momento de la infección. No obstante, hay que reconocer que las circunstancias pudieron ocurrir de forma diferente, es decir, el no poder saber con precisión el momento en que ocurrió la infección y si ésta fue precedida por el factor de exposición medido limita la capacidad de establecer una relación causal en forma satisfactoria. Por otro lado, las altas densidades de mosquitos encontradas en casi toda la ciudad, que suponen un alto nivel de exposición de la población, no permiten medir la fuerza de la asociación entre el uso de mosquiteros e insecticidas y la disminución del riesgo de infectarse, toda vez que con la información disponible no es posible conocer el sitio de la exposición al vector. Asimismo, tampoco permite precisar la frecuencia y momento en que los insecticidas se utilizan, ya que la picadura de un mosquito infectado no se realiza por fuerza en el interior de la vivienda.
Otro aspecto a considerar en la interpretación de los resultados se relaciona con la validez de las pruebas utilizadas para la determinación de anticuerpos en la población. Como requisito mínimo se esperaría que tuvieran la capacidad de detectar en forma confiable a los individuos positivos y negativos, con lo cual se eliminaría el problema de clasificar de manera errónea a la población estudiada.

Al respecto, se puede afirmar que las técnicas utilizadas en este trabajo se validaron ampliamente, y que la sensibilidad y especificidad de ambas pruebas las hace adecuadas para su uso poblacional. No obstante, aunque sin trascendencia para el propósito del presente trabajo, existen otras limitantes de la prueba como el hecho de no poder informar con precisión el momento en que la persona se infectó, así como el serotipo que causó la infección por dengue.

En cuanto a la prueba para leptospira, la posibilidad de una reacción cruzada impide asegurar que $20 \%$ de los individuos que presentó reacción al menos a dos serovariedades verdaderamente haya sufrido infección por éstas. Sin embargo, en los resultados de este estudio sólo se incluyeron aquellos que demostraron los títulos más altos.

En este sentido, un aspecto importante a considerar es la utilidad relativa de la información que brinda el entrevistado en relación con el antecedente de haber sufrido determinada infección, sobre todo cuando es factible la presencia de casos asintomáticos de la enfermedad o cuando coincide con otras infecciones que comparten características clínicas. ${ }^{42}$ La baja sensibilidad encontrada para este dato obliga a considerar los sesgos potenciales al momento de su interpretación.

Si bien es cierto que se encontró asociación de algunas variables, como factores de riesgo o protectores con la presencia de anticuerpos en ambas enfermedades, también lo es que las diferencias encontradas entre los individuos fueron mínimas y por lo tanto el exceso de riesgo o protección no fue tan amplio como se esperaba, y en algunos casos no fue significativo. Empero, se puede asegurar que la fortaleza de estas asociaciones pudo verse afectada por el alto grado de exposición que enfrenta la población de Jáltipan y las altas prevalencias encontradas en la mayor parte de la ciudad. No obstante, la plausibilidad biológica en las relaciones estudiadas, así como la consistencia de los resultados entre los individuos y con los reportes de estudios similares, lo sustentan.

\section{Conclusiones}

La información presentada permite comprobar la magnitud de la transmisión del dengue en el país y en el 
estado de Veracruz, así como el alto riesgo para la ocurrencia de brotes de dengue hemorrágico en algunas áreas del territorio nacional mexicano. Asimismo, permite confirmar la transmisión de la leptospirosis y las serovariedades que circulan en esta región. De manera adicional se comprueba la coexistencia de diferentes agentes infecciosos compartiendo el nicho ecológico y la posibilidad de ser infectado por uno o más de ellos. ${ }^{43}$ Esto resulta de particular importancia cuando la ocurrencia de estos padecimientos coincide en el tiempo y cuando, además, presentan características clínicas semejantes que implican un problema para el médico de primer contacto al momento de establecer un diagnóstico oportuno y el tratamiento apropiado. Estos hechos fundamentan la difusión de estos hallazgos para brindar elementos al personal médico que contribuyan a mejorar el diagnóstico y tratamiento de los enfermos.

Para finalizar, los resultados obtenidos corroboran el peligro de la aparición, reemergencia y diseminación de una gran variedad de enfermedades infecciosas en todo el planeta. ${ }^{3,4,44,45}$ Por tal motivo, es importante el desarrollo de estrategias integrales de vigilancia epidemiológica, prevención y control de estas enfermedades que se base en la participación conjunta del personal de salud, autoridades y la comunidad. ${ }^{8,46}$

\section{Agradecimientos}

Este trabajo fue evaluado por el Comité de Evaluación Ética Institucional y se realizó con apoyo del Fondo para el Fomento de la Investigación Médica (FOFOI) de la Coordinación de Investigación en Salud del Instituto Mexicano del Seguro Social, IMSS.

\section{Referencias}

I. Shope R. Global climate change and infectious diseases. Environ Health Perspect 1991;96:171-174.

2. Jetten TH, Focks DA. Potential changes in the distribution of dengue transmission under climate warming. Am J Trop Med Hyg 1997;57(3):285-297.

3. Gratz NG. Emerging and resurging vector-borne diseases. Annu Rev Entomol 1999;44:5।-75.

4. Guzmán MG, Kourí G, Pelegrino JL. Enfermedades virales emergentes. Rev Cubana Med Trop 200 I;53(I):5-15.

5. Rodhain F.The situation of dengue in the world. Bull Soc Pathol Exot 1996;89(2):87-90.

6. Pinheiro FP, Corber SJ. Global situation of dengue and dengue haemorrhagic fever, and its emergence in the Americas. Wld Hlth Statis Quart 1997;50:61-169.

7. El Dengue en Centroamérica: las epidemias del 2000. Boletín Epidemiológico. Organización Panamericana de la Salud 2000;2I (4):4-8.
8. Guzman MG, Kouri G. Dengue and dengue hemorrhagic fever in the Americas: lessons and challenges. J Clin Virol 2003;27(I): I- I3. 9. Gómez Dantés H, Ramos Bonifaz B, Tapia Conyer R. El riesgo de transmisión del dengue: un espacio para la estratificación. Salud Publica Mex 1995;37:88-97.

10. Koopman JS, Prevots DR, Vaca MMA, Gómez DH, Zárate AM et al. Determinants and predictors of dengue infection in Mexico. Am J Epidemiol 1991;133(II): I I68-I I78.

II. Herrera BE, Prevots DR, Zarate ML, Silva L, Sepulveda AJ. First reported outbreak of classical dengue fever at I,700 meters above sea level in Guerrero state, Mexico, June 1988. Am J Trop Med Hyg 1992;46(6):649-653.

12. Ibáñez-Bernal S, Gómez-Dantés H. Los vectores del dengue en México: una revisión crítica. Salud Publica Mex 1995;37:53-62.

I3. Fernández-Salas I, Flores-Leal A. El papel del vector Aedes aegypti en la epidemiología del dengue en México. Salud Publica Mex 1995;37:45-52.

14. Gómez DH. Monografía sobre la epidemiología del dengue.

Dirección General de Epidemiología. México: Secretaría de Salud, 1993.

15. Narro Robles J, Gómez Dantés H. El dengue en México: un problema prioritario de salud pública. Salud Publica Mex 1995;37:12-20. 16. Briseño-García B, Gómez-Dantes H, Argot-Ramírez E, Montesano R, Vázquez-Martínez Al, Ibáñez-Bernal $S$ et al. Potential risk for dengue hemorrhagic fever: the isolation of serotype dengue-3 in Mexico. Emerg Infect Dis 1996;2:133-135.

17. Rico-Hesse R, Harrison LM, Salas RA, Tovar D, Nisalak A, Ramos C, et al. Origins of dengue type 2 viruses associated with increased pathogenicity in the Americas. Virology 1997;230:244-25I.

18. Loroño-Pino MA, Farfan-Ale JA, Zapata-Peraza AL, Rosado-Paredes EP, Flores-Flores LF, García-Rejón JE et al. Introduction of the American/ Asian genotype of dengue 2 virus into the Yucatan State of México.Am J Trop Med Hyg 2004 Oct;7I (4):485-492.

19. Secretaría de Salud. Dirección General de Epidemiología. Boletines Epidemiológicos Anuales. México: Secretaría de Salud, 1985-2003. 20. Navarrete EJ,Vázquez MJL,Vázquez RJA, Gómez DH. Epidemiología del dengue y dengue hemorrágico en el Instituto Mexicano del Seguro Social. Rev Per Epidemiol 2002;7(I). Biblioteca Virtual en Salud de la Universidad Nacional Mayor de San Marcos. Disponible en: www.unmsm.edu.pe.

2I.Vado-Solís I, Marrufo-Cárdenas M, Jiménez-Delgadillo B, Alzina-López A, Laviada-Molina H, Suárez-Solís \ y Zavala-Velázquez J. Clinicalepidemiological study of leptospirosis in humans and reservoirs in Yucatan, Mexico. Rev Inst Med Trop Sao Paulo 2002;44(6):335-340. 22. Norma Oficial Mexicana para la Vigilancia Epidemiológica, Prevención y Control de las Enfermedades Transmitidas por Vector. NOM-032-SS2-2002.

23. Porter KR,Widjaja S, Lohita HD, Hadiwijaya SH, Maroef CN, Suharyono $\mathrm{W}$ et al. Evaluation of a commercially available immunoglobulin $\mathrm{M}$ capture enzyme-linked immunosorbent assay kit for diagnosting acute dengue infections. Clin Diagn Lab Immunol 1999;6(5):74I-744.

24.Tsai FT.Arboviruses. En: Murray PR, Baron EJ, Pfaller MA, Tenover FC, Yolken RH, ed. Manual of clinical microbiology. American Society of Microbiology. 7a. edición. Washington, DC:American Society of Microbiology Press, 1999:1 107-I 124.

25. Office internacional def epizooties. Manual of standard for diagnostic test vaccines. 2a. edición. París: OIE, 1992.

26. Coreño-Juárez $O$. Prevalencia de anticuerpos contra dengue en la zona huasteca de San Luis Potosí (tesis). Mexico: UNAM, 1991.

27. Guzmán-Tirado M, Kourí-Flores G, Bravo-González J, Silva L, Vázquez-Ramudo S. Encuesta serológica nacional a virus dengue. Cuba 1982. Rev Cubana Med Trop 1984;36:131-138.

28. Guzmán M, Kouri G, Bravo G, De la Hoz F, Soler M, Hernández D. Encuesta seroepidemiológica retrospectiva a virus dengue en los 
municipios de Cienfuegos y Palmira. Rev Cubana Med Trop 1989;4I(3):32I-332.

29. Guzmán MG, Kourí GP, Bravo J, Soler M,Vázquez S, Morier L. Dengue hemorrhagic fever in Cuba, 1981: a retrospective seroepidemiologic study. Am J Trop Med Hyg 1990;42(2): 179-184. 30. Ault SK. Environmental management: a re-emerging vector control strategy.Am J Trop Med Hyg 1994;50(6):35-49.

31.Ying Chk, Mei JCh, Shu MY.The predisposing and protective factors against dengue virus transmission by mosquito vector. Am J Epidemiol 1992;। 36(2):214-220.

32. Lloyd LS, Winch P, Ortega-Canto J, Kendall C. Results of a community-based Aedes aegypti control program in Merida, Yucatan, Mexico. Am J Trop Med Hyg 1992;46(6):635-642.

33. Winch P, Leontsini E, Rigau-Perez J, Ruiz-Perez M, Clark G, Gubler D. Community-based prevention programs in Puerto Rico: impact of knowledge, behavior and residential mosquito infestation. Am J Trop Med Hyg 2002;67(4):363-370.

34. Leal-Castellanos CB, Garcia-Suarez R, Gonzalez-Figueroa E, FuentesAllen JL, Escobedo-de la Peña J. Risk factors and the prevalence of leptospirosis infection in a rural community of Chiapas, Mexico. Epidemiol Infect 2003;13I(3): I |49-II56.

35. Katz A, Ansdell V, Efler P, Middleton Ch, Sasaki D. Leptospirosis in Hawaii, 1974-1998: epidemiologic analysis of 353 laborator-confirmed cases. Am J Trop Med Hyg 2002;66(I):6I-70.

36. Acha PN, Cifres B. Zoonosis y enfermedades transmisibles comunes al hombre y a los animales. 20 edición. Washington, DC: Organización Panamericana de la Salud, 1992;14-36.

37.Vado-Solís IA, Cárdenas-Marrufo MF, Laviada-Molina H,Vargas-Puerto F, Jiménez-Delgadillo B, Zavala-Velásquez JE. Estudio de casos clínicos e incidencia de leptospirosis humana en el estado de Yucatán, México, durante el periodo de 1998 a 2000. Rev Biomed 2002;13:157-164. 38. Zavala-Velázquez JE,Vado-Solís IA, Rodríguez-Félix MA, RodríguezAngulo EM, Barrera-Pérez MA, Guzmán-Marín ES. Leptospirosis anictérica en un brote epidémico de dengue en la península de Yucatán. Rev Biomed 1998;9:78-83.

39. Gavaldón GD, Cisneros AM, Rojas N, Moles-Cervantes LP. Importancia de la leptospirosis humana en México. Detección de anticuerpos antileptospira en una población de donadores de sangre. Gac Med Mex 1995; I3I(3):289-292.

40. Colín-Ortíz JR, Caballero-Servín A, Alarcón-Vital JA, Pérez-Serna JC, Bernal-Vélez C, Cuellar-Espinoza A. Estudios sobre leptospirosis en México (1961-1995). Higiene 1997;5: I 42-150.

41. Hernández AM. Garrido F, Salazar-Martínez E. Sesgos en estudios epidemiológicos. Salud Publica Mex 2000;42(5):438-446.

42. Sanders EJ, Rigau-Perez JG, Smits HL, Deseda CC, Vorndam VA, Aye T et al. Increase of leptospirosis in dengue negative patients after a hurricane in Puerto Rico in 1966.Am J Trop Med Hyg 1999; 61 (3):399-404. 43. Loroño-Pino MA, Cropp CB, Farfan JA, Vorndam AV,

Rodriguez-Angulo EM, Rosado-Paredes EP et al. Common ocurrence of concurrent infections by multiple dengue virus serotypes. Am J Trop Med Hyg 1999;6I(5):725-730.

44. Gubler DJ.Arboviruses as imported disease agents: the need for increased awareness. Arch Virol Suppl 1996; I I:2 I-32.

45. Kilbourne ED. New viral diseases. A real and potential problem without boundaries. JAMA 1990;264(I):68-70.

46. Gubler DJ. Dengue and dengue hemorrhagic fever. Clin Microbiol Rev 1998; I (3):480-496. 